\title{
A Cohort Study of Healthcare Utilization in Older Adults with Undiagnosed Dementia
}

\author{
Sei J. Lee, MD MAS ${ }^{1,2}$, Eric B. Larson, MD MPH ${ }^{3}$, Sascha Dublin, MD PhD ${ }^{3}$, Rod Walker, $M S^{3}$, \\ Zachary Marcum, PharmD PhD ${ }^{4}$, and Deborah Barnes, $P h D^{1,2}$ \\ 'University of California, San Francisco, San Francisco, CA, USA; ${ }^{2}$ San Francisco VA Health Care System, San Francisco, CA, USA; ${ }^{3}$ Group Health \\ Research Institute, Seattle, WA, USA; ${ }^{4}$ University of Washington, Seattle, WA, USA.
}

KEY WORDS: dementia; utilization.

$\mathrm{J}$ Gen Intern Med 33(1):13-5

DOI: $10.1007 / \mathrm{s} 11606-017-4162-3$

(c) Society of General Internal Medicine (outside the USA) 2017

\section{INTRODUCTION}

Dementia is common among older adults and is associated with high rates of healthcare utilization. ${ }^{1}$ However, studies suggest that up to half of patients with dementia remain undiagnosed. ${ }^{2}$ Our objective was to determine healthcare utilization rates among patients with undiagnosed dementia.

\section{METHODS}

We identified patients who developed dementia while enrolled in the Adult Changes in Thought (ACT) study, a prospective cohort study of older adults enrolled in Group Health $(\mathrm{GH})$, an integrated healthcare system. ACT participants are assessed for dementia every 2 years, with abnormal results leading to a detailed diagnostic evaluation. ${ }^{3}$ For each of the 980 ACT participants who were diagnosed with dementia between 1994 and 2014, we examined GH electronic health data in the 2 years prior to diagnosis in ACT to determine whether dementia was recognized by their healthcare providers before diagnosis within the ACT research study. If a dementia diagnosis, memory complaint or dementia medication was found in the participant's GH electronic health data prior to diagnosis in ACT, the participant was classified as having "diagnosed dementia" ( $n=544)$; otherwise, the participant was classified as having "undiagnosed dementia" $(n=436)$. For each undiagnosed case, we randomly selected three participants, matched on age, gender and date of ACT visit ( \pm 180 days), who did not develop dementia while in ACT to serve as our "no dementia" group $(n=1308)$.

The number of outpatient visits, "no-shows" for scheduled outpatient visits, emergency department visits and hospitalizations were obtained from $\mathrm{GH}$ electronic utilization data for the 2-year period before the index ACT study dementia screening visit. We describe characteristics and healthcare utilization patterns of

Published online August 28, 2017 participants with diagnosed dementia, undiagnosed dementia and no dementia over 2 years. We used ageadjusted conditional logistic regression to formally compare participants with undiagnosed dementia and those without dementia.

\section{RESULTS}

In terms of baseline characteristics, participants with undiagnosed dementia appear to represent an intermediate group between participants with diagnosed dementia and those with no dementia (Table 1). For example, $35 \%$ of participants with diagnosed dementia had cerebrovascular disease, compared to $22 \%$ of participants with undiagnosed dementia and $10 \%$ of participants with no dementia $(p<0.001$, undiagnosed vs. no dementia).

In terms of healthcare utilization, participants with undiagnosed dementia again appear to be an intermediate group between participants with diagnosed dementia and those with no dementia (Table 2). Participants with diagnosed dementia had 2.5 emergency department visits over 2 years, compared to 1.7 visits for undiagnosed participants and 0.8 visits for participants with no dementia $(p<0.001$, undiagnosed vs. no dementia).

We saw similar patterns when examining the proportion of participants with at least one visit. For example, 53\% of diagnosed dementia participants had at least one clinic "noshow" over 2 years, compared to $43 \%$ of undiagnosed dementia participants and $29 \%$ of no dementia participants ( $p<0.001$, undiagnosed vs. no dementia). Similarly, $46 \%$ of diagnosed dementia participants had at least one hospitalization, compared to $31 \%$ of undiagnosed participants and $21 \%$ of participants with no dementia $(p=0.02$, undiagnosed vs. no dementia).

\section{DISCUSSION}

Leveraging data from a large prospective cohort study of dementia embedded within an integrated healthcare system with excellent healthcare utilization data, we found that patients with undiagnosed dementia were an intermediate group between patients with diagnosed dementia (most 
Table 1 Baseline Characteristics of Patients with Undiagnosed Dementia and No Dementia

\begin{tabular}{|c|c|c|c|c|}
\hline & $\begin{array}{l}\text { Diagnosed dementia } \\
(n=544)\end{array}$ & $\begin{array}{l}\text { Undiagnosed dementia } \\
(n=436)\end{array}$ & $\begin{array}{l}\text { No dementia } \\
(n=1308)\end{array}$ & $\begin{array}{l}\text { Age-adjusted } \\
p \text {-value* }\end{array}$ \\
\hline \multicolumn{5}{|l|}{ Demographics } \\
\hline Age, years & 85.1 & 85.2 & 78.2 & N/A \\
\hline Female $(\%)$ & 64 & 61 & 59 & 0.16 \\
\hline Non-Hispanic white ${ }^{\dagger}(\%)$ & 91 & 92 & 90 & 0.75 \\
\hline \multicolumn{5}{|l|}{ Cardiovascular diagnoses $(\%)$} \\
\hline Hypertension & 63 & 58 & 51 & 0.73 \\
\hline Diabetes mellitus & 15 & 20 & 13 & 0.001 \\
\hline Tobacco use disorder & 7 & 5 & 8 & 0.89 \\
\hline Atrial fibrillation & 26 & 22 & 14 & 0.02 \\
\hline Myocardial infarction & 16 & 12 & 7 & 0.11 \\
\hline Congestive heart failure & 28 & 28 & 13 & 0.004 \\
\hline Cerebrovascular disease & 35 & 22 & 10 & $<0.001$ \\
\hline \multicolumn{5}{|c|}{ Psychiatric and neurologic diagnoses (\%) } \\
\hline Traumatic brain injury & 14 & 8 & 3 & 0.003 \\
\hline Psychoses & 21 & 7 & 1 & $<0.001$ \\
\hline Depression & 38 & 25 & 13 & $<0.001$ \\
\hline Anxiety & 19 & 11 & 8 & 0.05 \\
\hline \multicolumn{5}{|l|}{ Other diagnoses $(\%)$} \\
\hline Chronic lung disease & 21 & 20 & 19 & 0.47 \\
\hline Solid tumor w/o metastases & 23 & 20 & 21 & 0.04 \\
\hline Hypothyroidism & 22 & 19 & 11 & 0.04 \\
\hline
\end{tabular}

N/A not applicable

*P-values based on conditional logistic regression model with adjustment for age, comparing undiagnosed dementia and no dementia

${ }^{\prime}$ Two of the "undiagnosed dementia" patients and 10 of the "no dementia" patients were missing information on racelethnicity. Percentages provided here are among those not missing race/ethnicity

healthcare needs) and no dementia (least healthcare needs). Compared to patients without dementia, patients with undiagnosed dementia were more likely to "no-show" for scheduled appointments, to visit the emergency department and to be hospitalized.

One explanation for these findings is that patients with undiagnosed dementia have an intermediate comorbidity burden compared to patients with diagnosed dementia and those with no dementia, resulting in the observed intermediate healthcare utilization. However, previous studies have found that accounting for comorbidity burden did not fully explain higher healthcare utilization in dementia patients, ${ }^{4}$ suggesting there may be additional reasons why dementia patients may utilize more healthcare. Specifically, patients with undiagnosed dementia may be less able to access care due to forgotten appointments and increased "no-shows," resulting in the higher rates of emergency department visits and hospitalizations. Identifying patients with undiagnosed dementia may allow for targeting of support services to remind patients and caregivers about appointments. These reminders may reduce "no-shows" and lead to fewer emergency department visits and

Table 2 Two-Year Healthcare Utilization Patterns of Patients with Diagnosed Dementia, Undiagnosed Dementia and No dementia

\begin{tabular}{|c|c|c|c|c|}
\hline & $\begin{array}{l}\text { Diagnosed dementia } \\
(n=544)\end{array}$ & $\begin{array}{l}\text { Undiagnosed dementia } \\
(n=436)\end{array}$ & $\begin{array}{l}\text { No dementia } \\
(n=1308)\end{array}$ & $\begin{array}{l}\text { Age-adjusted } \\
p \text {-value* }\end{array}$ \\
\hline \multicolumn{5}{|c|}{ Total number of healthcare episodes per patient over 2 years: } \\
\hline Clinic visits & 14.5 & 11.8 & 9.8 & 0.08 \\
\hline Clinic "no-show" & 0.5 & 0.4 & 0.3 & $<0.001$ \\
\hline ED visits & 2.5 & 1.7 & 0.8 & $<0.001$ \\
\hline Hospitalizations & 5.2 & 2.6 & 1.7 & 0.09 \\
\hline \multicolumn{5}{|c|}{ Percentage $(\%)$ of patients with at least one: } \\
\hline Clinic visit & 99 & 99 & 99 & 0.97 \\
\hline Clinic "no-show" & 53 & 43 & 29 & $<0.001$ \\
\hline ED visit & 53 & 48 & 25 & $<0.001$ \\
\hline Hospitalization & 46 & 31 & 21 & 0.02 \\
\hline
\end{tabular}

Values are mean for continuous variables or percentage for categorical variables

ED emergency department

*P-values based on conditional logistic regression model adjusted for age, comparing undiagnosed to no dementia 
hospitalizations, which are often distressing for cognitively impaired patients and costly to the healthcare system. ${ }^{5}$

Acknowledgements: We would like to acknowledge the participants in the ACT study, who continue to help us learn more about older adults with cognitive impairment.

Corresponding Author: Sei J. Lee, MD MAS; University of California, San Francisco, San Francisco, CA, USA (e-mail: sei.lee@ucsf.edu). Contributors: All contributors are authors.

Funders: This project was supported by a pilot award from Health Care Systems Research Network (R24AG045050), a pilot award from Tideswell at UCSF, a pilot award from the UCSF Older Adults Independence Center (P3OAG044281) and the SD Bechtel, Jr. Foundation. Dr. Lee's effort was supported by RO1AG047897 and VA HSR\&D IIR 15434. This project was made possible through the facilities and resources of the San Francisco VA Health Care System.

\section{Compliance with Ethical Standards:}

Prior Presentations: Alzheimer's Association International Conference, 2017 (London).
Conflict of Interest: The authors declare that they do not have a conflict of interest.

\section{REFERENCES}

1. Feng Z, Coots LA, Kaganova Y, Wiener JM. Hospital and ED use among medicare beneficiaries with dementia varies by setting and proximity to death. Health Affairs. 2014;33(4):683-690.

2. Prince M BR, Ferri C. Executive Summary, World Alzheimer Report 2011: The Benefits of Early Diagnosis and Intervention. London: Institute of Psychiatry, King's College London;2011.

3. Kukull WA, Higdon R, Bowen JD, et al. Dementia and Alzheimer's disease incidence: A prospective cohort study. Archives of Neurology. 2002;59(11):1737-1746.

4. Zhao Y, Kuo TC, Weir S, Kramer MS, Ash AS. Healthcare costs and utilization for Medicare beneficiaries with Alzheimer's. BMC Health Serv Res. 2008;8:108

5. Shankar KN, Bhatia BK, Schuur JD. Toward patient-centered care: A systematic review of older adults' views of quality emergency care. Annals of Emergency Medicine. 2014;63(5):529-550.e521. 